\title{
Oil Red O (ORO) reagent for detection of latent fingermarks: a review
}

\author{
Gurvinder Singh Bumbrah $^{1^{*}}$ (D), Gurvinder Singh Sodhi ${ }^{2}$ and Jasjeet Kaur ${ }^{3}$
}

\begin{abstract}
Background: Fingerprints are most frequently used to establish the identity of a person in medicolegal cases. Wide range of methods (optical, physical, and chemical) can be used to detect latent fingerprints on porous and nonporous items recovered from crime scenes. Oil Red O, also called solvent red 27, is a lipophilic dye, which means that it stains fat and lipid components in biological samples. It is also used to stain oil and waxes to a red hue. Oil Red $\mathrm{O}$ is used to detect latent fingerprints on dry and wet porous items like paper and cardboard.

Result: The reagent develops clear, stable, and red-colored fingerprints which may be discerned in natural light. Conclusion: Although the physical developer can also lift latent impressions from wet porous surfaces, the method is a multistep one and requires immersion of delicate, paper-like articles in a sequence of working solutions. Compared to that, the operational steps of Oil Red $\mathrm{O}$ method are simple and cost effective and require less equipment to process items.
\end{abstract}

Keywords: Fingerprints, Latent impressions, Oil Red O, Porous item, Wet surfaces

\section{Background}

Fingerprints are the most infallible means of identification. To render visibility to latent fingerprints that are most frequently found at crime scenes, a host of methods (optical, physical, and chemical) may be used (Bumbrah 2016; Bumbrah et al. 2016; Bumbrah 2017). Oil Red O (ORO) is one of the chemical reagents which have the potential to detect fingermarks on dry and wet porous and semi-porous surfaces. It fixes the lipid components of the sweat residue (Beaudoin 2004). However, effectiveness of ORO reagent for processing latent impressions on non-porous surfaces is questionable.

\section{Main text}

Fingerprints are the most important individual evidence usually found on various artifacts lifted from the crime scenes. These provide a direct link to the suspect and are formed by deposition of sweat from pores on friction ridge skin of fingertips (Champod et al. 2016; Ramotowski 2012). Since sweat is colorless in nature, the impressions formed there from too

\footnotetext{
* Correspondence: bumbrah85@gmail.com

${ }^{1}$ Department of Forensic Science, Punjabi University, Patiala 147002, India Full list of author information is available at the end of the article
}

are invisible and hence termed as latent fingerprints (Ramotowski 2012; Thomas 1978).

Three types of glands, viz. eccrine, apocrine, and sebaceous, are responsible for natural secretions from fingertips. Eccrine glands are widely distributed throughout the body and are particularly numerous on the palms of hands and the soles of feet. These glands produce sweat that is more than $98 \%$ water. Several other inorganic and organic chemicals are secreted by these glands as a result of general metabolism and catabolism (Knowles 1978; Kuno 1934). Eccrine sweat consists of proteins, urea, amino acids, uric acid, lactic acid, sugars, creatinine, and choline while sebaceous sweat consists of glycerides, fatty acids, wax esters, squalene, and sterol esters (Scruton et al. 1975).

The chemical components of sweat residue may be broadly classified into two classes: water-soluble and water-insoluble (Cantu et al. 2007). The amino acids and inorganic ions, such as sodium, potassium, and chloride, belong to the water-soluble class. Ninhydrin (NIN) and 1,8-diazafluoren-9-one (DFO) methods are used to target these water-soluble components of latent fingermark residue. The water-insoluble class includes proteins, lipids, and fats. This water-insoluble class is further subdivided into two fractions: robust and labile. Robust 
fraction includes proteins and lipo-proteins, while labile fraction includes saturated and unsaturated fatty acids, triglycerides, and lipids. The labile components undergo rapid chemical transformations upon exposure to air. However, these components are not affected by their exposure to water. The robust components form strong hydrogen bond with cellulose content of paper and are, therefore, retained on its surface for long periods (Ramotowski 2012; Salma et al. 2008). ORO is used to target labile fraction, and physical developer (PD) is used to target robust fraction of latent fingermarks (Cantu 2001; Salma et al. 2008). Figure 1 displays the classification scheme to process different fractions of latent fingermarks.

Different kinds of optical, physical, and/or chemical methods are routinely used to visualize latent fingermarks. Optical methods are nondestructive in nature and utilize electromagnetic radiation of appropriate wavelengths to render visibility to latent prints. Physical methods involve the physical interaction with sweat deposition of latent fingermarks. Chemical methods can be used to develop the latent fingermarks by chemically converting a particular constituent of sweat into a colored derivative. These methods may be used in isolation or in concert with others to enhance the visibility of developed prints. Selection of the method depends on nature (porous, semi-porous, nonporous), color (light, dark, multicolored), texture (smooth and rough), and condition (wet or dry) of surface on which the latent fingermark is impinged (Bumbrah 2016; Bumbrah et al. 2016; Bumbrah 2017; Champod et al. 2016; Ramotowski 2012).

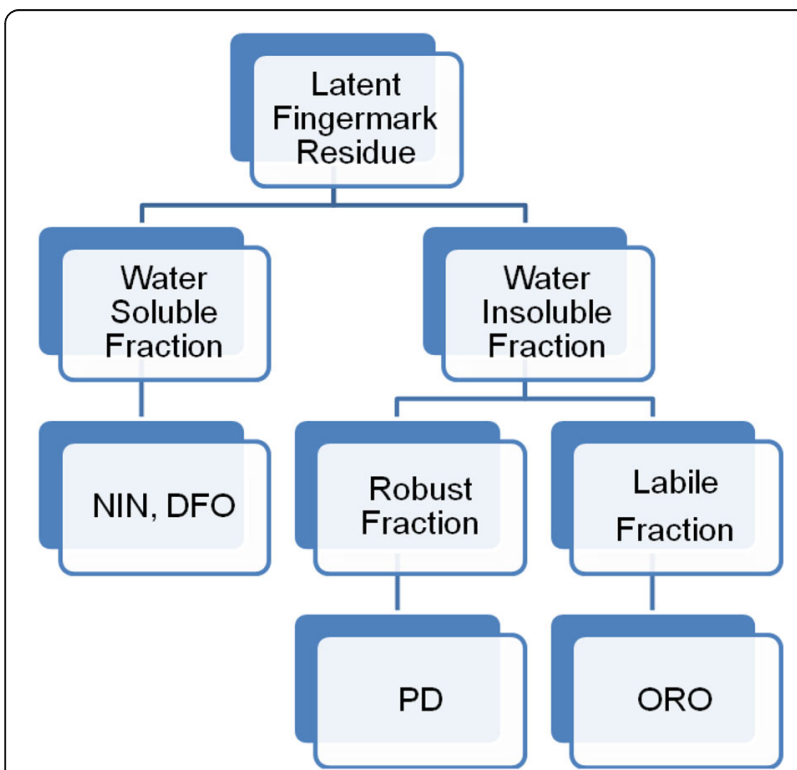

Fig. 1 Classification scheme to process different fractions of latent fingermarks
Oil Red O (ORO) is a lipophilic dye. It was first introduced as a reagent for the detection of latent fingermarks in 2004, although it has been used in biological staining techniques since the late 1920 s to stain lipid material in tissue sections (Beaudoin 2004; French 1926; Proescher 1927). It is a lysochrome (soluble lipid stain) used for detecting lipo-proteins separated by electrophoresis. ORO received its first reported forensic application in 2002 for the use of visualizing lip prints (Ali et al. 2005; Castello et al. 2002). ORO is a fat-soluble diazo dye and dissolves in lipids and stains them to a red hue. It interacts with the labile fraction of latent fingermark residues (Salma et al. 2008). ORO method is used when amino acids content in latent fingermarks is less and when porous surface is wet or exposed to high level of humidity (Beaudoin 2004).

\section{The reagent}

ORO is a fat-soluble, lipid sensitive, diazo $\left(\mathrm{R}-\mathrm{N}=\mathrm{N}-\mathrm{R}^{\prime}\right)$ dye. Empirical formula of ORO is $\mathrm{C}_{26} \mathrm{H}_{24} \mathrm{~N}_{4} \mathrm{O}$. Molecular weight of ORO is 408.510 (Beaudoin 2004). ORO contains two azo groups $(-\mathrm{N}=\mathrm{N}-)$, four methyl groups $\left(-\mathrm{CH}_{3}\right)$ (attached to two benzene rings) and one hydroxyl group $(-\mathrm{OH})$ (attached to one naphthalene ring). The structure of ORO is depicted in Fig. 2. Azo dyes have structural confirmations that prevent them from ionizing and thus facilitating their solubility in lipids. ORO absorbs radiation having wavelength of $518 \mathrm{~nm}$ and stains the item red (Guigui and Beaudoin 2007). Azo group and aromatic ring (as chromophore) are responsible for absorption of radiation and hence the color. ORO has hydroxyl auxophore and the red stain is attributed to it (Ali et al. 2005; Guigui and Beaudoin 2007). ORO interacts with fragile, relatively short-lived "labile fraction" of latent fingermarks whereas PD reagent interacts with stable, long-lived "robust fraction" latent fingermarks (Cantu 2001; Salma et al. 2008).

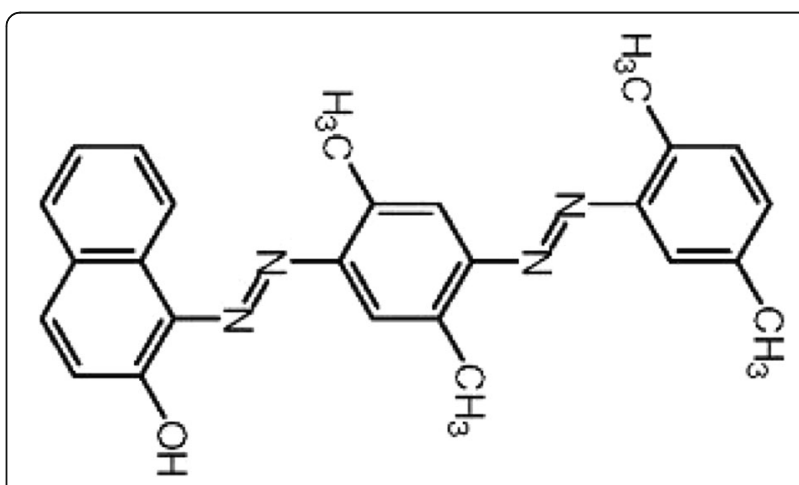

Fig. 2 Structure of Oil Red O (ORO) 


\section{Methodology}

ORO staining process is simple and requires only three steps: coloration, neutralization, and drying. Two test solutions (stain solution and buffer solution) are required for developing latent fingermarks on dry or wet porous surfaces by ORO reagent. Stain solution is required to stain the latent fingermarks while buffer solution is required to maintain the $\mathrm{pH}$. This buffer is used to neutralize the basic nature of ORO (Beaudoin 2011). The buffer solution neutralizes the alkaline content of the coloring solution to make the treated objects stable and secure (Beaudoin 2004).

\section{Stain solution}

It is prepared in the following steps:

- The ORO reagent $(1.54 \mathrm{~g})$ is dissolved in methanol (770 ml).

- A solution of sodium hydroxide $(9.2 \mathrm{~g})$ in water (230 $\mathrm{ml})$ is prepared.

- The aqueous solution of sodium hydroxide is added to the alcoholic solution of ORO.

- The contents are thoroughly mixed and filtered (using a standard filter paper).

- The filtrate is stored in a dark brown bottle away from light (Beaudoin 2004, 2011).

The stain solution, which is kept in dark atmosphere, remains stable for about 8 months, even though the color of stain solution changes to orange-yellow after 2 months (Beaudoin 2011; Salma et al. 2008).

\section{Buffer solution}

It is prepared in the following steps:

- Dissolve sodium carbonate (26.5 g) in water (2.0 l) and shake till it completely solubilizes.

- Add concentrated nitric acid (70\%; $18.3 \mathrm{ml})$ to sodium carbonate solution in small lots and with constant stirring.

- Dilute the contents by adding water $(2.5 \mathrm{l})$ and store in a dark-colored bottle as shown in Fig. 3 (Beaudoin 2004; Salma et al. 2008).

Another buffer solution may be prepared as follows:

- Dissolve sodium phosphate monobasic monohydrate $\left(\mathrm{NaH}_{2} \mathrm{PO}_{4} . \mathrm{H}_{2} \mathrm{O} ; 101.5 \mathrm{~g}\right)$ in distilled water (1.0 l).

- Dissolve sodium phosphate dibasic heptahydrate $\left(\mathrm{Na}_{2} \mathrm{HPO}_{4} \cdot 7 \mathrm{H}_{2} \mathrm{O} ; 339.0 \mathrm{~g}\right)$ in distilled water (1.0 l).

- Mix the two solutions and dilute with distilled water (41) (Beaudoin 2011).

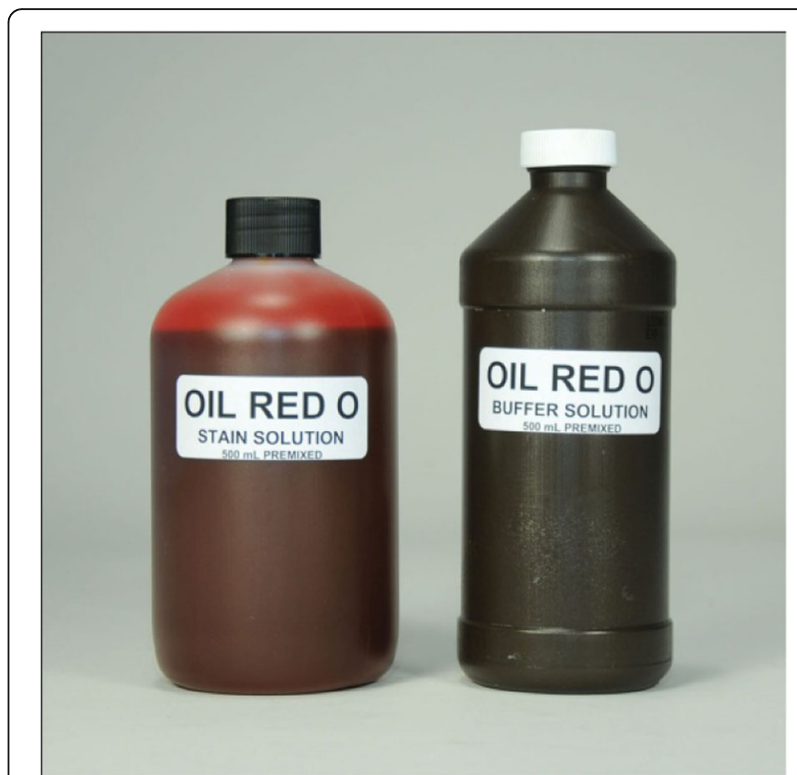

Fig. 3 The stain and buffer solutions for Oil Red O processing

This buffer is more stable and has more strength than the previous one.

\section{Test procedure}

Latent fingermarks can be developed on dry or wet porous surfaces by following the sequence of steps:

\section{Staining}

- The article bearing the latent impressions is immersed in the stain solution and agitated (using titer plate shaker) for about $60 \mathrm{~min}$. A shaker which is normally used for this purpose is shown in Fig. 4. The tray in which the article is agitated is normally covered with a paraffin film to avoid loss of methanol due to evaporation. Article must be constantly immersed in solution (Beaudoin 2011).

\section{Buffer solution}

- The article is removed from the stain solution and drained.

- It is then immersed in the buffer solution for few seconds to adjust the $\mathrm{pH}$. The buffer solution neutralizes the alkaline content absorbed on to the item as a result of its immersion in the stain solution. Removal of the alkali from the surface stabilizes the developed prints.

\section{Drying}

- The surface is rinsed in distilled water and allowed to dry under natural conditions. 


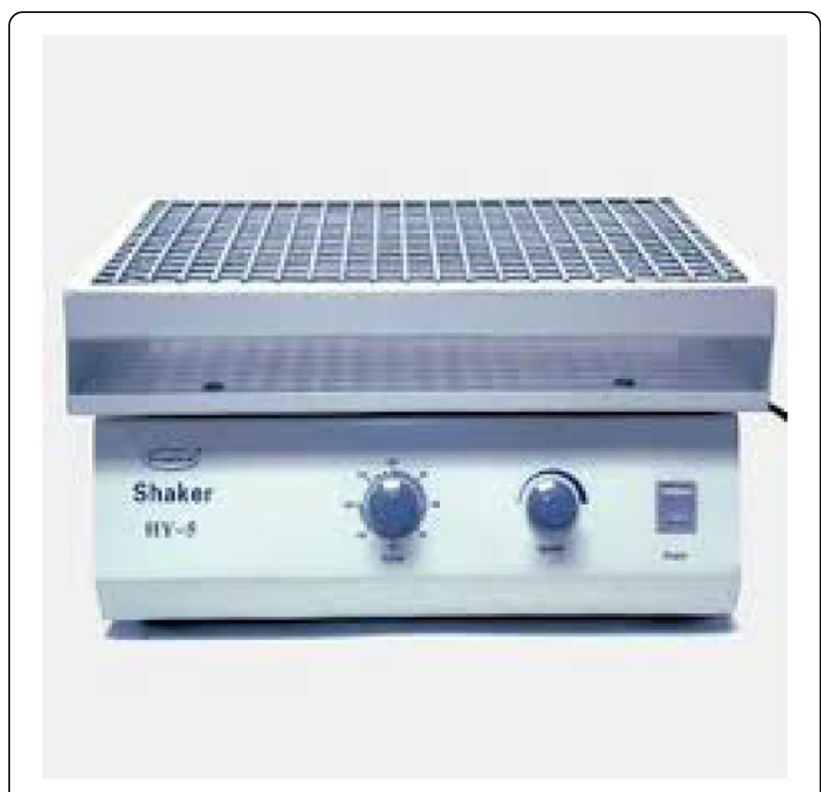

Fig. 4 A shaker is generally used to agitate the contents

- Drying process may be accelerated by heating the article in an oven at $50^{\circ} \mathrm{C}$. Red colored fingermarks develop on pink background (Beaudoin 2004, 2011; Salma et al. 2008).

Another method to prepare and use stain solution is as follows: Dissolve the ORO reagent $(0.05 \mathrm{~g})$ in propylene glycol $(100 \mathrm{ml})$ and warm $\left(95^{\circ} \mathrm{C}\right)$ with constant stirring. Allow the contents to cool to room temperature, and remove any residual ORO by vacuum filtration (Frick et al. 2012). Alternatively, filter it through a funnel fitted with fine wire mesh (Honig and Yoak 2016). Store the stain solution at room temperature in Schott bottles wrapped in aluminum foil. The items bearing the latent fingerprints are placed in glass trays and immersed in ORO reagent ( $15 \mathrm{~min})$, with manual agitation provided by gentle rocking the tray for $30 \mathrm{~s}$ at the beginning of treatment. After development, ORO-treated samples are rinsed twice in a deionized water bath under running water and were air dried on paper towels at room temperature (Frick et al. 2012). Honig and Yoak suggested the use of running deionized water in place of buffer solution as later did not influence the quality of developed prints and cost of the method can be significantly reduces (Honig and Yoak 2016).

ORO staining solution could be reused for treating new batch of evidences if solution has a reddish-burgundy color. If color of ORO solution changes to orange-yellow, then it cannot be used because amount of the test reagent becomes insufficient for optimum detection of latent fingermarks. It was observed that as long as methanol did not evaporate, stain solution does not deteriorate (Beaudoin
2011). Shelf life of ORO solution was reported to be up to 1 year (Guigui and Beaudoin 2007).

A chemical reagent which fixes the labile fraction of sweat residue for developing latent fingermarks should not be dissolved in non-polar solvents because these solvents will wash away the labile components before these have a chance to interact with the developing reagent. It is for this reason that ORO is dissolved in methanol-water mixture. On coming in contact with the labile components of sweat residue, the stain partitions between the original solvent mixture, in which it is less soluble and the fats/lipids, in which it is more soluble. The molecules of ORO get preferentially dissolved in fat and lipid components and impart red color to these biomolecules (Kutt and Tsaltas 1959). The preferential solubility of ORO in fats and lipids is a physical phenomenon which is controlled by thermodynamic considerations. The entropy of the system increases as the molecules of ORO become dispersed in the lipophilic media. Entropy is a measure of disorderness in a closed system. The greater the degree of disorderness, the greater is the positive value of entropy and more stable is the system. When ORO becomes dissolved in fats and lipids, its molecules become randomized in the sweat residue, thereby increasing the entropy. The fallout is that the red color imparted to the fingermark residue is more intense than that of the original stain. The outcome is that clear, red-colored ridges become visualized on pink background (Beaudoin 2004; Salma et al. 2008). Figure 5 shows the fingermark developed with ORO on white paper.

\section{Evaluation of Oil Red 0 method}

Use of methanol or propylene glycol-based ORO formulations over PD was advocated for developing fresh (less than 1 week old) and aged (7 days old) charged and sebum-rich latent fingermarks on wet papers (Frick et al. 2012). However, the use of PD was suggested for developing older and uncharged latent fingermarks. It was

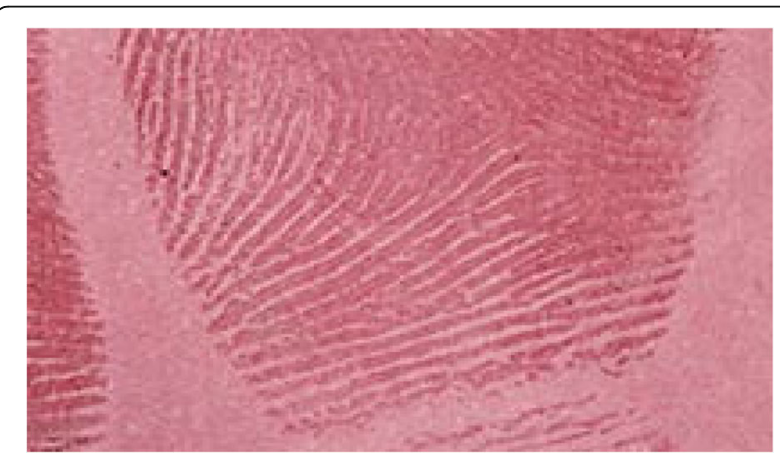

Fig. 5 Fingermark developed with ORO on white paper 
observed that use of propylene glycol in ORO formulation improves the sensitivity of reagent along with the contrast and quality of developed prints in a short time of period. Propylene glycol-based ORO formulation is simple and economical to prepare than methanol-based ORO formulation. Staining involved the immersion of article in ORO (15 min), with gentle manual agitation $(30 \mathrm{~s})$ at the beginning of the development time (Frick et al. 2012).

Salama and co-workers observed that ORO interacts with variety of water-soluble and water-insoluble components of latent fingermark deposits (Salma et al. 2008). The quality of developed prints depends on immersion time in water and age of latent finger impression. Beaudoin reported a case in which ORO method was used to detect and develop latent fingermarks on a 21-year-old paper (Beaudoin 2011). Immediate processing of white paper with ORO was recommended to maximize the chances of success. It was observed that the quality of developed prints depends on the nature of surface (porous, semi-porous, non-porous), method applied, and time interval between deposition and processing of latent fingermarks (Boudreault and Beaudoin 2017). In addition to it, the quality of developed prints depends on the pore size of the paper which subsequently depends on the manufacturing process and chemical composition of the paper (Rawji and Beaudoin 2006). It was noted that latent fingermarks remain stable for longer period of time on semi-porous and non-porous surfaces than porous surfaces. The quality of prints developed, on white paper, with ORO method degraded with passage of time (Boudreault and Beaudoin 2017). ORO does not involve the use of alternate light source, and results will not fade after development (Rawji and Beaudoin 2006). The use of commercial available fingermark simulants (as quality control standards) was not recommended for the assessment of latent fingermarks developing reagents like PD, ORO, NIN, and 1,2-indanedione (IND) (Zadnik et al. 2013). It was observed that sebaceous-rich marks survived longer than eccrine-rich marks in field conditions than laboratory conditions. Use of field conditions to examine the longevity of submerged marks was recommended (Sutton et al. 2014). Water treatment of surface before deposition of fingermarks produced poorer results as compared to water treatment after deposition of fingermarks (Rawji and Beaudoin 2006).

ORO reagent develops optimum quality fingermarks on different varieties of papers including kraft paper and glossy and rough side of corrugated brown cardboard (Beaudoin 2004). Although the background surface in some cases was stained pink, the red-colored ridges could be easily discerned. However, the reagent failed to develop prints on non-porous items like glass. The use of ORO for developing aged prints (less than 30 days) on wet, porous surfaces including thermal paper was suggested (Honig and Yoak 2016; Rawji and Beaudoin 2007; Salma et al. 2008; Simmons et al. 2014). Latent fingermarks impinged on thermal papers, followed by immersion in water for $2 \mathrm{~h}$, could be developed even after 30 days with ORO. Likewise, imprints deposited on photocopier paper, followed by soaking in water for $24 \mathrm{~h}$, could be developed after a lapse of 30 days. Prints could also be developed by ORO method on unplasticized polyvinyl chloride that has remained soaked in water for week (Wood and James 2009a, 2009b).

It has been pertinent to compare the performance of ORO method with the PD technique which too develops fingermarks on dry and wet porous surfaces. Neither ORO nor PD develops fresh or aged (7 days old) latent fingermarks on paper which were submerged in petrol (Wood and James 2009a, 2009b). With ORO reagent, the calligraphic script on paper, no doubt, gets despoiled, yet the overall results were better than those obtained with PD. Further, although ORO produced better results on photocopier paper than on kraft paper, the reverse was true of PD. The use of ORO was recommended to develop latent fingermarks on moist, porous surfaces (thermal and white standard paper) after comparing it with PD method (Rawji and Beaudoin 2006).

ORO was found to be less damaging to evidence and provides better quality prints than PD (Guigui and Beaudoin 2007). Operational setup of ORO has been much less complex than PD and provides high-quality intense prints, and therefore, it could be used as an alternative to the traditional PD (Beaudoin 2004; Guigui and Beaudoin 2007; Rawji and Beaudoin 2006; Salma et al. 2008). The superiority of ORO over PD was advocated to develop fresh and aged (less than 30 days) latent fingermarks on wet, porous surfaces including white and thermal papers (Beaudoin 2011; Guigui and Beaudoin 2007; McMullen and Beaudoin 2013; Mingchao and Danhua 2015). In contrast to this, other authors observed that ORO was not an effective method for developing latent fingermarks as thermal paper loses writing or information on treating with ORO (Rawji and Beaudoin 2006).

ORO outperforms PD so long that the latent impressions were fresh or at the most 4 weeks old. For more aged impressions, PD performs better. No doubt, ORO method has a fewer steps as compared to PD technique, yet the immersion time $(90 \mathrm{~min})$ in the stain solution make the overall process slower. Nevertheless, ORO technique may be used after all routine amino acid-specific reagents have been tried out without success (Guigui and Beaudoin 2007; Salma et al. 2008). For fresh prints, the recommended order of sequencing was 
ORO, followed by PD (Guigui and Beaudoin 2007; Rawji and Beaudoin 2007).

Simmons and co-workers compared different processing methods (PD, ORO, modified PD) to develop latent fingermarks (fresh and aged) on moist porous surfaces (white paper, cardboard) (Simmons et al. 2014). Tween 20 in place of Synperonic-N in modified PD composition was used for enhancing latent fingermarks. The use of PD and modified PD over ORO for processing such types of substrates was suggested. In an another comparative study, Honig and Yoak suggested that ORO could be used as an efficient alternative to PD and NIN for enhancing latent fingermarks on dry and moist, porous surfaces as it provides intense and better quality prints than PD and NIN method (Honig and Yoak 2016).

PD was recommended over ORO to develop aged (older than 28 days) latent fingermarks on different kinds of papers (Ramotowski 2012). A number of studies have demonstrated that ORO was superior to PD; however, such studies used "loaded" sebum rich fingermarks (Salma et al. 2008; Rawji and Beaudoin 2006). In contrast, other studies using "ungroomed" fingermarks found that ORO did not outperform PD for the detection of latent fingermarks up to 7 days old (Wood and James 2009a, 2009b).

Wood and James observed that neither PD nor ORO develop quality fingermarks on card surfaces when using normal fingermarks as opposed to loaded fingermarks (Wood and James 2009a, 2009b). Less effectiveness of ORO towards old prints, insensitivity towards watersoluble components of latent fingermarks, and negligible success towards developing latent fingermarks on non-porous surfaces are some of the major drawbacks of ORO reagent method.

\section{Post-treatment procedures}

Sutton and co-workers recommended the use of Sudan black and gentian violet over ORO for developing fresh and aged (more than 10 days old) latent fingermarks on smooth, non-porous surfaces like glass, plastic, and metal (Sutton et al. 2014). Beaudoin suggested the use of ORO followed by rhodamine $6 \mathrm{G}$ for the development of fresh and aged (10 days old) latent fingermarks on dark, moist, and absorbent substrates such as black cardboard (Beaudoin 2012).

\section{Sequence studies}

For wet, porous surfaces, the recommended sequence was ORO followed by PD while for dry, porous surfaces, the recommended sequence was $\mathrm{DFO} \rightarrow \mathrm{NIN} \rightarrow$ ORO $\rightarrow$ PD (Beaudoin 2011). Use of sequential processing with propylene glycol-based ORO and Tween 20-based PD formulations over propylene glycol-based ORO formulation alone was advocated for developing fresh and aged latent fingermarks on white copy papers. It was observed that both methods can be used as complementary as both methods targets the different components of latent fingermarks (Frick et al. 2017). In another study, sequential processing with NIN followed by ORO was recommended to develop latent fingermarks on wet, thermal papers (McMullen and Beaudoin 2013). In a similar study, sequential processing with DFO followed by NIN followed by ORO was suggested to develop latent fingermarks on wet papers (Guigui and Beaudoin 2007; McMullen and Beaudoin 2013). PD could be successfully used after processing the items with ORO as ORO did not influence the quality of prints developed by PD (Swofford 2010). Use of PD followed by ORO was suggested for developing latent fingermarks as ORO enhances the quality of prints developed by PD (Salma et al. 2008). McMullen and Beaudoin demonstrated that the sequential processing with DFO-NINORO did not prevent the development of useful fingermarks on paper and ORO developed fingermarks that had been undetected with the DFO-NIN sequence (McMullen and Beaudoin 2013). ORO enhances the quality of prints already developed with NIN and DFO. However, sequential processing with NIN and DFO after ORO reduces the contrast produced by ORO. ORO was more sensitive than NIN but less sensitive than DFO. The order in which these methods were applied was selected so that the success of each technique was not hindered by a preceding technique.

The insertion of ORO in fingerprint development sequence was advocated. The use of ORO reagent before $\mathrm{PD}$ was suggested as ORO treatment did not interfere in subsequent processing. The recommended sequence for processing latent fingermarks on dry paper was DFO $\rightarrow$ $\mathrm{NIN} \rightarrow \mathrm{ORO} \rightarrow$ PD while the sequence $\mathrm{ORO} \rightarrow \mathrm{PD}$ was recommended for processing latent fingermarks on wet paper. ORO was less damaging to evidence and gives better quality prints than PD (Guigui and Beaudoin 2007).

The inclusion of propylene glycol-based ORO reagent method into the processing sequence after treatment with IND and followed by treatment with PD (IND $\rightarrow$ modified ORO reagent $\rightarrow$ PD) was suggested for the development of fresh latent fingermarks on wet paper as it increases the efficiency of sequence in addition to quality of developed prints and no interference was observed between these techniques, as each technique targeted a different component of latent fingermarks (Frick et al. 2013).

\section{Conclusion}

Oil Red $\mathrm{O}$ is an effective and efficient method to develop latent fingermarks on dry and wet porous surfaces present at crime scene. The present technique is simple, fast, cost-effective, and non-destructive in nature and 
requires less equipment to process items. It develops red-colored fingerprints on pink background. The quality of developed prints is up to the mark, provided that the impressions are revealed within a span of 4 weeks. For prints which are more than 4 weeks old, the PD method is more effective than ORO. Standardized testing of ORO method should be conducted to evaluate its efficiency and reliability in developing moist latent fingermarks on different surfaces. More research is required to better understand the reaction mechanism involved and to improve the sensitivity of this for processing latent fingermarks (aged and degraded) on difficult and unusual surfaces.

\section{Abbreviations}

DFO: 1,8-Diazafluoren-9-one; IND: 1,2-Indanedione; NIN: Ninhydrin; ORO: Oil Red O; PD: Physical developer

\section{Acknowledgements}

Authors acknowledge the Head of Department of Forensic Science, Punjabi University, Patiala, for her support and encouragement.

\section{Funding}

None.

Availability of data and materials

Related literature is available to corresponding author.

\section{Authors' contributions}

All authors have contributed equally in the designing and completion of the study as well as in preparation of manuscript. All authors read and approved the final manuscript.

\section{Ethics approval and consent to participate}

Not applicable.

\section{Consent for publication}

Not applicable.

\section{Competing interests}

The authors declare that they have no competing interests.

\section{Publisher's Note}

Springer Nature remains neutral with regard to jurisdictional claims in published maps and institutional affiliations.

\section{Author details}

${ }^{1}$ Department of Forensic Science, Punjabi University, Patiala 147002, India. ${ }^{2}$ Forensic Science Unit, S.G.T.B. Khalsa College, University of Delhi, Delhi 110007, India. 'Shaheed Rajguru College of Applied Sciences for Women, University of Delhi, Vasundhara Enclave, Delhi 110096, India.

Received: 8 February 2018 Accepted: 18 December 2018 Published online: 05 January 2019

\section{References}

Ali MF, El Ali BM, Speight JG (2005) Handbook of industrial chemistry - organic chemicals. McGraw Hill, New York, pp 259-288

Beaudoin A (2004) New technique for revealing latent fingerprints on wet, porous surfaces: oil red O. J For Ident 54(4):413-421

Beaudoin A (2011) Oil red O: fingerprint development on a 21-year old cold case. J For Ident 61(1):50-59

Beaudoin A (2012) Fingerprint staining technique on dark and wetted porous surfaces: oil red $\mathrm{O}$ and rhodamine 6G. J For Ident 62(4):315-329

Boudreault A, Beaudoin A (2017) Pseudo-operational study on the efficiency of various fingermark development techniques during the aging process. J Forensic Ident 67(1):85-117
Bumbrah GS (2016) Small particle reagent (SPR) method for detection of latent fingermarks: a review. Egypt J Forensic Sci 6(4):328-332

Bumbrah GS (2017) Cyanoacrylate fuming method for detection of latent fingermarks: a review. Egypt J Forensic Sci 7(1):4-11

Bumbrah GS, Sharma RM, Jasuja OP (2016) Emerging latent fingerprint technologies: a review. Research Reports Forensic Med Sci 6:39-50

Cantu A, Burow D, Wilson J (2007) On some properties of the oil red O fingerprint visualization reagent. International Fingerprint Research Group Meeting, Canberra 26-30 March

Cantu AA (2001) Silver physical developers for the visualization of latent prints on paper. Forensic Sci Rev 13(1):29-64

Castello A, Alvarez M, Miquel M, Verdu F (2002) Long-lasting lipsticks and latent prints. For Sci Comm 4

Champod C, Lennard C, Margot P, Stoilovic M (2016) Fingerprints and other ridge skin impressions, 2nd edn. CRC Press, Boca Raton, pp 211-212

French RW (1926) Fat stains. Stain Tech 1:79

Frick AA, Fritz P, van Bronswijk W, Beaudoin A, Bleay S, Lennard C, Lewis SW (2017) Investigations into the influence of donor traits on performance of fingermark development reagents. Part 2: oil red O and PD. J Forensic Ident 67(3):427-446

Frick AA, Fritz P, Lewis SW, van Bronswijk W (2012) A modified oil red O formulation for the detection of latent fingermarks on porous substrates. J For Ident 62(6):623-641

Frick AA, Fritz P, Lewis SW, van Bronswijk W (2013) Sequencing of a modified oil red $O$ development technique for the detection of latent fingermarks on paper surfaces. J For Ident 63(4):369-385

Guigui K, Beaudoin A (2007) The use of oil red O in sequence with other methods of fingerprint development. J For Ident 57(4):550-581

Honig M, Yoak J (2016) Oil red O: a comparative performance study. J For Ident 66(2):118-133

Knowles AM (1978) Aspects of physicochemical methods for the detection of fingerprints. J Phys E: Sci Instrum 11(8):713-721

Kuno Y (1934) The physiology of human perspiration. J. \& A. Churchill Ltd., London, pp 1-22

Kutt $H$, Tsaltas $T$ (1959) Staining properties of oil red $\mathrm{O}$ and a method of the partial purification of the commercial product. Clin Chem 5(2):149-160

McMullen L, Beaudoin A (2013) Application of oil red O following DFO and ninhydrin sequential treatment: enhancing latent fingerprints on dry, porous surfaces. J For Ident 63(4):387-423

Mingchao W, Danhua W (2015) Evaluation of fingerprints developed by physical developer and oil red O on paper surface. Forensic Sci Tech 40(4):283-287

Proescher F (1927) Oil red O pyridin: a rapid fat stain. Stain Tech 2(2):60-61

Ramotowski R (2012) Lee and Gaensslen's advances in fingerprint technology, 3rd edn. CRC Press, Boca Raton, Florida, pp 85-89

Rawji A, Beaudoin A (2006) Oil red O versus physical developer on wet papers: a comparative study. J For Ident 56(1):33-54

Rawji A, Beaudoin A (2007) Oil red O versus physical developer for wet substances: a comparative study. Identification Canada 30:4-18

Salma J, Aumeer-Donovan S, Lennard C, Roux C (2008) Evaluation of the fingermark reagent oil red $\mathrm{O}$ as a possible replacement for physical developer. J For Ident 58(2):203-237

Scruton B, Robins BW, Blott BM (1975) The deposition of fingerprint films. J Phys D Appl Phys 8(6):714-723

Simmons RK, Deacon P, Farrugia KJ (2014) Water soaked porous evidence: a comparison of processing methods. J For Ident 64(2):157-173

Sutton R, Grenci C, Hrubesova L (2014) A comparison on the longevity of submerged marks in field and laboratory conditions. J For Ident 64(2):143-156

Swofford HJ (2010) The efficacy of commercial vs. noncommercial physical developer solutions and sequential enhancement of friction ridge impressions using potassium iodide. J Forensic Ident 60(1):19-33

Thomas GL (1978) The physics of fingerprints and their detection. J Phys E: Sci Instrum 11(8):722-731

Wood MA, James T (2009a) Latent fingerprint persistence and development techniques on wet surfaces. Fingerprint Whorld 35:90-100

Wood MA, James T (2009b) ORO: the physical developer replacement. Sci Justice 49(4):272-276

Zadnik S, van Bronswijk W, Frick AA, Fritz P, Lewis SW (2013) Fingermark simulants and their inherent problems: a comparison with latent fingermark deposits. J Forensic Ident 63(5):593-608 